\title{
Morphometrics Characterization of Thin-Tail Sheep in Lowland and Highland Areas
}

\author{
Depison $^{\mathrm{a}, *}$, W. P. B. Putra ${ }^{\mathrm{b}}$, Gushairiyanto ${ }^{\mathrm{a}}$, , Y. Alwi $^{\mathrm{a}}$, \& H. Suryani ${ }^{\mathrm{a}}$ \\ aFaculty of Animal Science, Jambi University \\ Jambi-Muara Bulian Rd. Km 15, Muaro Jambi, Jambi, Indonesia 36122 \\ ${ }^{b}$ Research Center for Biotechnology, Indonesian Institute of Science \\ Bogor-Jakarta Rd. Km 46, Cibinong, Bogor, West Java, Indonesia 16911 \\ *Corresponding author: depison.nasution@unja.ac.id \\ (Received 07-10-2020; Revised 19-03-2021; Accepted 07-04-2021)
}

\begin{abstract}
Breed characterization in the livestock is important for the breeding program in the future. This research aimed to characterize Thin-tail sheep in the highland and lowland areas of Jambi Province based on 7 body measurements and body indices using multivariate analysis. Data were collected from 160 sheep consisted of 80 rams and 80 ewes from each area (320 sheep in total with 1-2 years old). The research showed that the principal component analysis (PCA) in this study was explained $65.84 \%-72.30 \%$ by body measurements and $78.23 \%-84.99 \%$ by body indices of the total phenotypic variance of animals. The body measurement of cannon circumference (CC) and body indices of area index (AI), relative cannon index (RCI), dactyl thorax index (DTI), conformation index (CI), and index of body weight (IBW) were selected as the discriminating variable for Thin-tail sheep in different areas. However, this study's canonical correlation (rc) values were 0.44 for body measurements and 0.47 for body indices. Therefore, about $67.5 \%$ of Thin-tail sheep from the lowland area and $57.5 \%$ of Thin-tail sheep from the highland area can be characterized with body measurements. Hence, about $61.2 \%$ of Thin-tail sheep from the lowland area and $65.6 \%$ of Thin-tail sheep from the highland area can be characterized with body indices. Temperature, humidity, rainfall, and length of radiation in the highland areas are lower than in the lowland areas. The cluster analysis in four Thin-tail sheep populations revealed two clusters, i.e., cluster 1 consisted of Kerinci and Sungai Penuh and cluster 2 consisted of Muaro Jambi and Batanghari. It was concluded that about $60 \%$ of Thin-tail sheep could be characterized through their body indices.
\end{abstract}

Keywords: thin-tail sheep; characterization; principal component; canonical correlation; cluster

\section{INTRODUCTION}

Thin-tail sheep are local Indonesian sheep kept by smallholders for meat production. Sheep are livestock that are beneficial for human interests, namely as a food source for animal protein. The average weaning weight of Thin-tail lambs in Sedan Village was $8.53 \pm 1.57 \mathrm{~kg}$ (Najmuddin \& Nasich, 2019). The average body weight for one year old male sheep was $27.834 \pm 6.914 \mathrm{~kg}$ and female $22.798 \pm 2.823 \mathrm{~kg}$ (Ashari et al., 2015). In addition, the average litter size and lambing interval in Thin-tail ewes were $1.82 \pm 0.42 \mathrm{lamb} / \mathrm{ewe} / \mathrm{year}$ and $9.66 \pm 0.69$ months, respectively (Najmuddin \& Nasich, 2019). One of the breeding tracts for Thin-tail sheep in Indonesia is located in Jambi Province. The total number of sheep in this province in 2017 reached 76,370 heads or $0.46 \%$ of the total sheep population $(16,462,274$ heads) in Indonesia (KEMENTAN RI, 2017).

One of the breeding tracts of Thin-tail sheep in Indonesia locates in lowland and highland areas of Jambi Province. The morphometric characterization of livestock is important for planning improvement, sustainable utilization, conservation strategies, and breed- ing programs (FAO, 2012). In small-ruminant animals, morphometric characterization can be performed with body measurements and body indices (Esquivelzeta et al., 2011; Ouchene-Khelifi et al., 2018; Putra \& Ilham, 2019; Markovic et al., 2019). Moreover, previous studies have classified the small-ruminant animals from different populations based on their morphometrics (Zaitoun et al., 2005; Traore et al., 2008; Nafti et al., 2014; Dekhili et al., 2014; Gatew et al., 2015; Hosseini et al., 2016; Birhanie et al., 2019; Jarquin et al., 2019).

Recently, the morphometrics characterization in livestock can be performed using three statistical analyses of principal component analysis (PCA), canonical discriminant analysis (CDA), and hierarchical cluster analysis (HCA). Moreover, these statistical analyses are widely used to characterize small-ruminant breeds from different populations (Birteeb et al., 2012; Aziz and Al-Hur, 2013; Boujenane et al., 2016; Dauda et al., 2018; N'Goranet al., 2019; Nunes et al., 2020).

Morphometric characterization can be carried out on body measurements, including body length, chest girth, wither height, rump height, chest depth, canon-bone length, canon-bone circumference, pelvic 
width, rump length, and rump width (Getahun et al., 2020; Josiane et al., 2020). Morphometrics can also be used to estimate body weight (Sabbioni et al., 2020), livestock selection, and preservation (Ashifudin et al., 2017). Morphometric data are needed for livestock identification, prediction of production potential, livestock productivity (Hilmawan et al., 2019; Mahmudi et al., 2019), continuity of breeding and livestock breeding (Saputra et al., 2019; Markovic et al., 2019). Several morphometric studies have been carried out on sheep to improve genetic quality based on morphometric data (Vazic et al., 2017; Salvagno \& Albarella, 2017; Sanni et al., 2018; Markovic et al., 2019). Linear Body Parameters and Craniometric Analysis in Etawah-Grade goats were used to predict prolificacy (Mulyono et al., 2018). However, there is no information regarding the morphometric characteristics of Thin-tail sheep in two different areas, namely the lowland and the highland areas. It is thought that different land-area conditions can affect the morphometric characteristics of livestock. This study aimed to characterize the morphometric characteristics of thin-tail sheep raised in the lowland and highland areas. The results of this study will be used as a basis for planning improvement, sustainable utilization, conservation strategies, conservation strategies, and breeding programs for thin-tail sheep in the highland area and lowland area in the future.

\section{MATERIALS AND METHODS}

\section{Research Site and Animals}

This research was conducted in the lowland area $(0-100 \mathrm{~m}$ asl) and in the highland area ( $>500 \mathrm{~m}$ asl) of Jambi Province of Indonesia locating at latitude $0^{\circ} 45^{\prime}-2^{\circ} 45^{\prime} \mathrm{S}$ and longitude $101^{\circ} 10^{\prime}-104^{\circ} 55^{\prime}$ E. (Central Bureau of Statistic, Jambi Province, 2021). Hence, the research sites of the highland area consisted of Sungai Penuh City (Kumun Debai Subdistrict and Tanah Kampung Subdistrict) and Kerinci Regency (Air Hangat Subdistrict and Depati Tujuh Subdistrict). Meanwhile, the research sites of the lowland area consisted of Muaro Jambi Regency (Sekernan Subdistrict and Kumpeh Subdistrict) and Batanghari Regency (Muaro Tembesi Subdistrict and Pemayung Subdistrict). The climatic conditions in the study areas were presented in Table
1. Therefore, the research sites of this study were illustrated in Figure 1.

A total of 320 Thin-tail sheep were collected from two areas of lowland (160 sheep) and highland (160 sheep) with a sex proportion of 80 rams and 80 ewes for each area. The sheep-raising system in both locations was the same, namely, the sheep were released in the morning and in the afternoon, they were kept in captivity.

\section{Management of Animal}

The lowland sheep and highland sheep were reared by the farmers with a semi-extensive farming system. The maintenance system applied was the experimental sheep were released in the morning and in the evening without being given forage or concentrates in the cage. The mating system was also occurred naturally.

\section{Data Collection}

The body measurements were taken from animals in a standing position with a raised head. Body measurements of animals were performed using a measuring stick and flexible measuring tape and taken based on the guideline of FAO (2012). Therefore, the weighing scale was used to obtain body weight (BW) of animals.

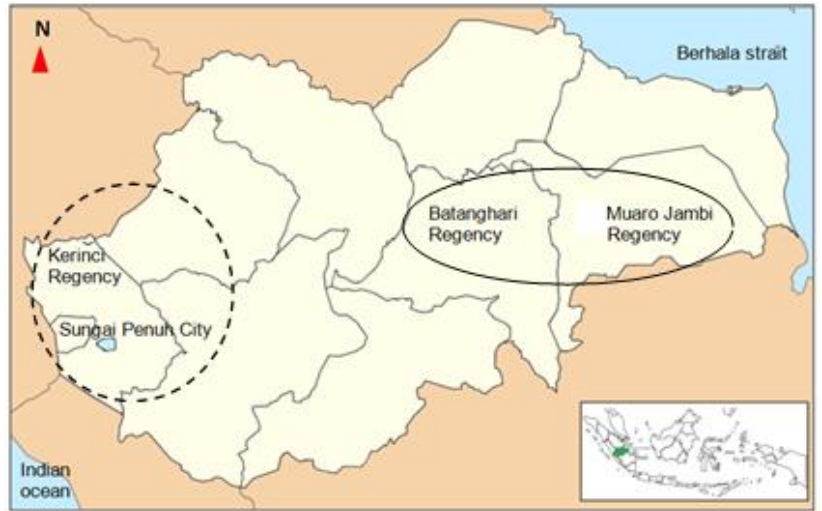

Figure 1. The Thin-tail sheep population in Jambi Province of Indonesia spread at lowland area (Batanghari Regency and Muaro Jambi Regency) and highland (Kerinci Regency and Sungai Penuh City) areas.

Table 1. Climatic condition of the study area

\begin{tabular}{|c|c|c|c|c|}
\hline \multirow{2}{*}{ Description } & \multicolumn{2}{|c|}{ Highland } & \multicolumn{2}{|c|}{ Lowland } \\
\hline & Sungai Penuh City & Kerinci Regency & Batanghari Regency & Muaro Jambi Regency \\
\hline Temperature $\left({ }^{\circ} \mathrm{C}\right)$ & 22.50 & 22.80 & 26.80 & 26.80 \\
\hline Wind speed (m/sec.) & 4.30 & 4.00 & 5.42 & 5.42 \\
\hline Humidity (\%) & 82.00 & 82.00 & 85.00 & 85.00 \\
\hline Air pressure (mb) & 923.89 & 923.89 & 1009.50 & 1009.50 \\
\hline Rainy day & 190.00 & 190.00 & 207.00 & 207.00 \\
\hline Sun ilumination (\%) & 57.00 & 57.00 & 116.97 & 124.58 \\
\hline Rainfall (mm) & $2.052,80$ & $2.052,80$ & 2.500 .00 & $2.500,00$ \\
\hline Regional high (asl) & $>1000$ & $>1000$ & 20 & 25 \\
\hline
\end{tabular}

Source: Central Bureau of Statistic, Jambi Province year 2019. 
Eight body measurements consisted of body length (BL), withers height $(\mathrm{WH})$, chest girth $(\mathrm{CG})$, chest depth (CD), shoulder width (SW), rump height $(\mathrm{RH})$, rump width (RW), and cannon circumference (CC). Body length (BL) was measured from the point of the shoulder to the pin bone. Wither height $(\mathrm{WH})$ was measured from the surface of a platform on which an animal stands to the withers of the animal. Chest girth (CG) was measured as the body circumference just behind the forelegs. Chest depth (CD) was measured from the most dorsal point of the withers to the ventral surface of the sternum. Shoulder width (SW) was measured as a distance from the left to the right shoulder blade. Rump height (RH) was measured from the surface of a platform to the rump. Rump width (RW) was measured as a distance between two tuber coxae. The scheme of body measurements in Thin-tail sheep was presented in Figure 2. Moreover, calculation of body indices were obtained in this study according to Birteeb et al. (2014), Khargharia et al. (2015), and Boujenane et al. (2015) as follow: Length index $(\mathrm{LI})=[\mathrm{BL} / \mathrm{WH}] \times 100$, Thoracic index $(\mathrm{TI})=[\mathrm{SW} /$ $\mathrm{CD}] \times 100$, Depth index $(\mathrm{DI})=[\mathrm{CD} / \mathrm{WH}] \times 100$, Height index $(\mathrm{HI})=[\mathrm{WH} / \mathrm{RH}] \times 100$, Thoracic development $(\mathrm{TD})=$ $[\mathrm{CG} / \mathrm{WH}] \times 100$, Dactyl thorax index $(\mathrm{DTI})=[\mathrm{CC} / \mathrm{CG}] \times 100$, Conformation index $(\mathrm{CI})=\mathrm{CG}^{2} / \mathrm{WH}$, Relative cannon index $(\mathrm{RCI})=[\mathrm{CC} / \mathrm{WH}] \times 100$, Index of body weight $(\mathrm{IBW})=[\mathrm{BW} / \mathrm{WH}] \times 100$, Body index $(\mathrm{BI})=[\mathrm{BL} / \mathrm{CG}] \times 100$, Proportionality $(\mathrm{Pr})=[\mathrm{WH} / \mathrm{BL}] \times 100$, Area index $(\mathrm{AI})=$ $\mathrm{WH} \times \mathrm{BL}$.

According to DTI value, the body of sheep can be described into four categories, i.e., light animals (DTI $<10.5)$, intermediary animals $(10.6<\mathrm{DTI}<10.8)$, light meat-type animals $(10.9<\mathrm{DTI}<11.0)$, and heavy meattype animals (DTI $>11.0)$. According to the $B I$ value, the body of sheep can be described into three categories, i.e., short or brevigline animals $(\mathrm{BI}<85)$, medigline animals $(86<\mathrm{BI}<88)$, and longline animals $(\mathrm{BI}>88)$ types (Esquivelzeta et al., 2011; Chacon et al., 2011).

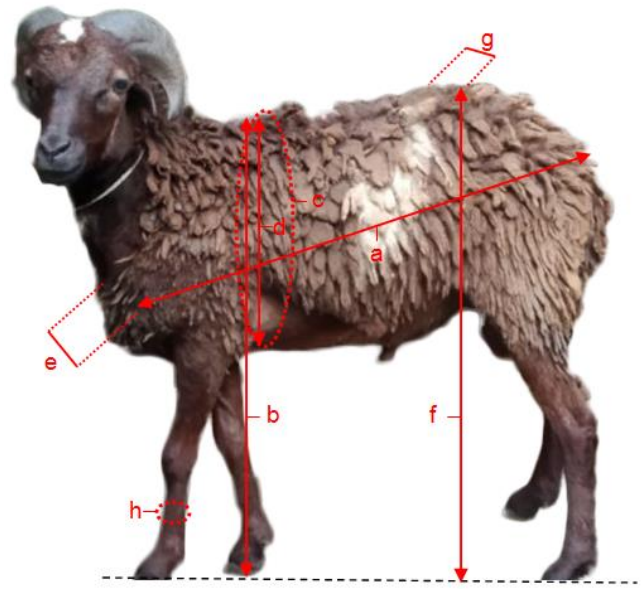

Figure 2. Body measurements scheme in Thin-tail sheep. a. body length (BL), b. withers height (WH), c. chest girth (CG), d. chest depth (CD), e. shoulder width (SW), f. rump height (RH), g. rump width (RH), h. cannon circumference (CC).

\section{Statistical Analysis}

The statistics parameter of mean, standard deviation, and Pearson's correlation coefficient (r) for body measurements and body indices were calculated with the SPSS 16.0 computer program. Meanwhile, the morphometric characterization in sheep was performed using three statistical analyses of principal component anaysis (PCA), canonical discriminant analysis (CDA), and hierarchical cluster analysis (HCA) using a similar computer program. The PCA was used to define the underlying structure among the variables in the analysis (Yunusa et al., 2013). The CDA was used to classify an observation, or several observations, into already known groups (Asamoah-Boaheng \& Sam, 2016). The HCA aimed to separate cases / objects into several groups that have different characteristics (Oliveira et al., 2018). In the PCA, Kaiser-Meyer-Olkin (KMO) measures of sampling adequacy and Bartlett's test of sphericity and communality were computed as the test validity. The KMO statistics vary between 0 and 1 . The value close to 0 indicates that there are large partial correlations compared to the total of correlations. A value close to 1 indicates that the sampling is appropriate. It was possible to accept a measure of sampling adequacy greater than 0.50 . The varimax criterion of the orthogonal rotation method was employed in the rotation of the factor matrix to enhance the interpretability of the factor analysis. In CDA, Mahalanobis distance $\left(D^{2}\right)$, tolerance $(T)$, Wilk's lambda $(\lambda)$ values and linear discriminant function were computed to obtain the discriminating variable for thin-tail sheep at two different areas. Here CDA was applied with the backward stepping automatic elimination method, with $F$ value entry $=3.84$ and $F$ value removal= 2.71. The $\mathrm{T}$ value (0 to 1$)$ was computed to detect the level of correlation among variables in the discriminant function. If a variable is highly correlated with one or more of the others, the $\mathrm{T}$ value is very small and the resulting estimates of the discriminant function coefficients may be unstable. The HCA was used to cluster Thin-tail sheep from different populations. The HCA in this study was performed using combination data (body weight, body measurements, and body indices) with the nearest neighbor method, Euclidean distance measure, and transform the value of $\mathrm{Z}$ score, counted by the following formula:

$$
Z=(x-\mu) / \sigma
$$

where $\mathrm{x}$ was the observed value (raw score), $\mu$ was population mean, $\sigma$ was the standard deviation of the population, and Z was Z Score (Standard Value).

\section{RESULTS}

\section{Animal Morphometrics}

Table 1 shows that the temperature, humidity, rainfall, and duration of radiation in the highland areas are lower than in the lowland areas. The morphometrics of Thin-tail sheep is presented in Table 2. The averages of 
BW, BL, WH, CG, SW, RH, and RW of Thin-tail population at highland areas were higher than those in lowland areas $(p<0.05)$. Meanwhile, the body indices of Thin-tail population at lowland areas and highland areas were

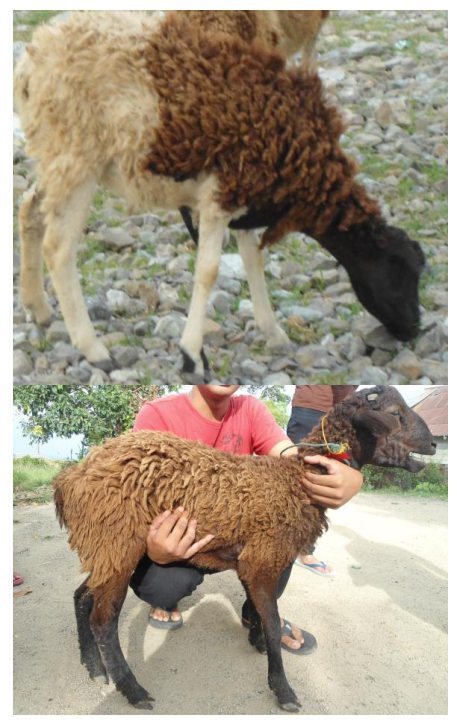

Thin-tail sheep in the highlands

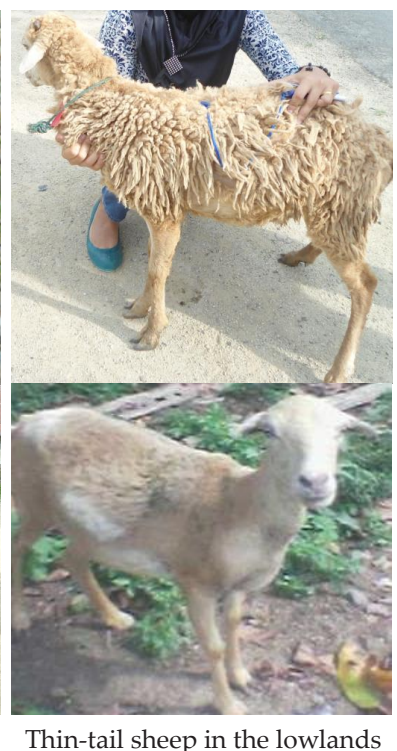

Thin-tail sheep in the lowlands
Figure 3. Thin-tail sheep in the highlands and lowlands in Jambi Province (The sheep in the highlands have various colors, in the lowlands generally light brown). not significantly different. However, the eight body indices of LI, TI, DI, TD, CI, RCI, IBW, and Pr in sheep in highland areas were higher than those in sheep in lowland areas. Commonly, the body weight, body measurements, and body indices in rams were higher than in ewes in each area. Nevertheless, the body indices of HI between rams and ewes in highland areas were similar.

\section{Phenotypic Correlation}

The correlation values among BW and body measurements ranged from 0.16 (BW-CG) to 0.65 (BW-WH) for thin-tail sheep at lowland areas and 0.34 (BW-RW) to 0.65 (BW-CG) for thin-tail sheep at highland areas (Table $3)$. Therefore, the r-value among BW and body indices (without IBW) ranged from -0.38 (BW-TI) to 0.08 (BWCI) for thin-tail sheep at lowland areas and -0.03 (BW$\mathrm{LI})$ to 0.64 (BW-AI) for thin-tail sheep at highland areas (Table 4).

The grasses consumed by thin-tail sheep at the lowland areas are generally A. Compresssus, O. Nodosa, L. Hexandra, and C. Dactylon with averages chemical compositions, namely Crude Protein $9.33 \%$, Crude Fiber $30.70 \%$, Crude fat $1.44 \%$, and Ash $10.55 \%$ (Table 5). In the highland areas, thin-tail sheep generally consumed the grasses of L. hexandra, B. muticum, D. ciliaris, O. nodosa, and E. colona, with averages chemical compositions of Crude Fiber $10.12 \%$, Crude Fiber $29.61 \%$, Crude fat $1.63 \%$, and Ash $10.30 \%$ (Table 5).

Table 2. The average of morphometric traits in Thin-tail sheep at two different areas

\begin{tabular}{|c|c|c|c|c|c|c|}
\hline \multirow{2}{*}{ Variables } & \multicolumn{3}{|c|}{ Lowland } & \multicolumn{3}{|c|}{ Highland } \\
\hline & $\operatorname{Ram}(\mathrm{N}=80)$ & Ewe $(\mathrm{N}=80)$ & Total $(\mathrm{N}=160)$ & $\operatorname{Ram}(\mathrm{N}=80)$ & Ewe $(\mathrm{N}=80)$ & Total $(\mathrm{N}=160)$ \\
\hline Body weight (kg) & $16.46 \pm 3.27$ & $15.25 \pm 2.33$ & $15.86 \pm 2.89^{a}$ & $18.85 \pm 2.44$ & $16.23 \pm 3.21$ & $17.54 \pm 3.13^{\mathrm{b}}$ \\
\hline \multicolumn{7}{|l|}{ Body measurements $(\mathrm{cm})$} \\
\hline Body length & $50.43 \pm 5.01$ & $48.37 \pm 4.52$ & $49.40 \pm 4.87^{a}$ & $53.26 \pm 4.40$ & $49.94 \pm 5.31$ & $51.60 \pm 5.14^{\mathrm{b}}$ \\
\hline Wither height & $51.63 \pm 4.20$ & $49.15 \pm 2.71$ & $50.39 \pm 3.74^{a}$ & $53.76 \pm 4.13$ & $50.57 \pm 4.49$ & $52.16 \pm 4.59^{\mathrm{b}}$ \\
\hline Chest girth & $54.61 \pm 14.80$ & $51.28 \pm 3.71$ & $52.4 \pm 10.89^{a}$ & $56.73 \pm 3.34$ & $53.64 \pm 4.51$ & $55.18 \pm 4.25^{\mathrm{b}}$ \\
\hline Chest depth & $23.74 \pm 2.35$ & $21.60 \pm 4.09$ & $22.67 \pm 3.49$ & $24.13 \pm 1.97$ & $23.27 \pm 4.27$ & $23.70 \pm 3.34$ \\
\hline Shoulder width & $11.54 \pm 1.10$ & $10.51 \pm 1.09$ & $11.02 \pm 1.21^{\mathrm{a}}$ & $13.00 \pm 0.80$ & $10.68 \pm 1.25$ & $11.84 \pm 1.56^{\mathrm{b}}$ \\
\hline Rump height & $53.33 \pm 4.79$ & $51.26 \pm 3.72$ & $52.30 \pm 4.40^{\mathrm{a}}$ & $56.00 \pm 3.70$ & $52.79 \pm 5.34$ & $54.40 \pm 4.85^{\mathrm{b}}$ \\
\hline Rump width & $12.71 \pm 2.05$ & $11.81 \pm 1.21$ & $12.26 \pm 1.74^{\mathrm{a}}$ & $14.19 \pm 2.78$ & $12.57 \pm 1.47$ & $13.38 \pm 2.36^{\mathrm{b}}$ \\
\hline Cannon circumferance & $6.89 \pm 0.61$ & $5.98 \pm 0.51$ & $6.44 \pm 0.72$ & $7.80 \pm 0.54$ & $6.53 \pm 0.32$ & $7.17 \pm 0.78$ \\
\hline \multicolumn{7}{|l|}{ Body indices } \\
\hline Length index & $97.72 \pm 6.06$ & $98.40 \pm 6.55$ & $98.06 \pm 6.30$ & $99.26 \pm 6.73$ & $98.94 \pm 7.77$ & $99.10 \pm 7.25$ \\
\hline Thoracic index & $48.99 \pm 5.95$ & $49.42 \pm 6.53$ & $49.20 \pm 6.23$ & $54.05 \pm 3.21$ & $46.63 \pm 6.57$ & $50.34 \pm 6.35$ \\
\hline Depth index & $46.07 \pm 4.03$ & $43.89 \pm 6.95$ & $44.98 \pm 5.77$ & $45.11 \pm 4.63$ & $46.05 \pm 7.16$ & $45.58 \pm 6.03$ \\
\hline Height index & $97.13 \pm 7.26$ & $96.27 \pm 7.79$ & $96.70 \pm 7.52$ & $96.02 \pm 4.27$ & $96.19 \pm 7.59$ & $96.10 \pm 6.14$ \\
\hline Thoracic development & $106.24 \pm 29.30$ & $104.36 \pm 5.60$ & $105.30 \pm 21.05$ & $105.92 \pm 7.37$ & $106.40 \pm 8.32$ & $106.16 \pm 7.84$ \\
\hline Dactyl thorax index & $26.55 \pm 13.62$ & $23.12 \pm 2.79$ & $24.84 \pm 9.95$ & $24.99 \pm 4.56$ & $23.57 \pm 3.21$ & $24.28 \pm 3.99$ \\
\hline Conformation index & $62.03 \pm 28.64$ & $53.65 \pm 5.65$ & $57.84 \pm 21.00$ & $60.15 \pm 6.15$ & $57.23 \pm 7.48$ & $58.69 \pm 6.98$ \\
\hline Relative cannon index & $24.67 \pm 3.99$ & $24.03 \pm 2.14$ & $24.35 \pm 3.21$ & $26.51 \pm 5.34$ & $24.99 \pm 3.16$ & $25.75 \pm 4.44$ \\
\hline Index of body weight & $31.78 \pm 5.15$ & $30.95 \pm 3.83$ & $31.36 \pm 4.54$ & $35.12 \pm 4.21$ & $31.95 \pm 4.88$ & $33.54 \pm 4.82$ \\
\hline Body index & $102.72 \pm 42.32$ & $94.65 \pm 9.49$ & $98.68 \pm 30.84$ & $94.00 \pm 7.32$ & $93.48 \pm 10.08$ & $93.74 \pm 8.78$ \\
\hline Proportionality & $2620.02 \pm 442.89$ & $2384.71 \pm 338.33$ & $2502.37 \pm 410.19$ & $2874.97 \pm 413.47$ & $2540.73 \pm 462.93$ & $2707.85 \pm 468.53$ \\
\hline Area index & $102.72 \pm 6.37$ & $101.99 \pm 5.73$ & $102.36 \pm 6.05$ & $101.20 \pm 6.83$ & $101.61 \pm 7.05$ & $101.41 \pm 6.92$ \\
\hline
\end{tabular}

$\mathrm{N}$ : number of animals. Means in the same row with different superscripts differ significantly $(\mathrm{p}<0.05)$ 
Table 3. Person's correlations between body weight and body measurements of Thin-tail sheep at lowland (above diagonal) and highland (under diagonal) areas

\begin{tabular}{lccccccccc}
\hline Body measurements & BW & BL & WH & CG & CD & SW & RH & RW & CC \\
\hline Body weight (BW) & - & $0.61^{* *}$ & $0.65^{* *}$ & $0.16^{*}$ & $0.55^{* *}$ & $0.23^{* *}$ & $0.64^{* *}$ & $0.48^{* *}$ & $0.42^{* *}$ \\
Body length (BL) & $0.55^{* *}$ & - & $0.73^{* *}$ & 0.09 & $0.43^{* *}$ & $0.18^{* *}$ & $0.47^{* *}$ & $0.43^{* *}$ & $0.32^{* *}$ \\
Wither height (WH) & $0.64^{* *}$ & $0.69^{* *}$ & - & $0.24^{* *}$ & $0.51^{* *}$ & $0.28^{* *}$ & $0.66^{* *}$ & $0.43^{* *}$ & $0.40^{* *}$ \\
Chest girth (CG) & $0.65^{* *}$ & $0.51^{* *}$ & $0.62^{* *}$ & - & $0.18^{* *}$ & 0.06 & $0.27^{* *}$ & $-0.26^{* *}$ & $0.24^{* *}$ \\
Chest depth (CD) & $0.49^{* *}$ & $0.36^{* *}$ & $0.35^{* *}$ & $0.40^{* *}$ & - & $0.22^{* *}$ & $0.46^{* *}$ & $0.43^{* *}$ & $0.39^{* *}$ \\
Shoulder width (SW) & $0.54^{* *}$ & $0.39^{* *}$ & $0.40^{* *}$ & $0.40^{* *}$ & $0.25^{* *}$ & - & $0.23^{* *}$ & $0.47^{* *}$ & $0.38^{* *}$ \\
Rump height (RH) & $0.57^{* *}$ & $0.55^{* *}$ & $0.81^{* *}$ & $0.53^{* *}$ & $0.31^{* *}$ & $0.39^{* *}$ & - & $0.39^{* *}$ & $0.49^{* *}$ \\
Rump width (RW) & $0.34^{* *}$ & $0.37^{* *}$ & $0.23^{* *}$ & $0.36^{* *}$ & $0.32^{* *}$ & $0.55^{* *}$ & $0.23^{* *}$ & - & $0.39^{* *}$ \\
Cannon circumferance (CC) & $0.53^{* *}$ & $0.43^{* *}$ & $0.45^{* *}$ & $0.43^{* *}$ & $0.25^{* *}$ & $0.73^{* *}$ & $0.41^{* *}$ & $0.44^{* *}$ & - \\
\hline
\end{tabular}

Note: ${ }^{*}(\mathrm{p}<0.05) ;{ }^{* *}(\mathrm{p}<0.01)$

Table 4. Person's correlations between body weight and body indices of Thin-tail sheep at lowland (above diagonal) and highland (under diagonal) areas

\begin{tabular}{|c|c|c|c|c|c|c|c|c|c|c|c|c|c|}
\hline Body indices & BW & LI & TI & DI & $\mathrm{HI}$ & TD & DTI & $\mathrm{CI}$ & $\mathrm{RCI}$ & IBW & $\mathrm{BI}$ & $\operatorname{Pr}$ & $\mathrm{AI}$ \\
\hline Body weight (BW) & - & $0.16^{*}$ & $-0.38^{*}$ & $0.27^{* *}$ & -0.09 & -0.10 & 0.14 & 0.08 & 0.04 & $0.92^{* *}$ & $0.18^{*}$ & $0.17^{*}$ & $-0.67^{* *}$ \\
\hline Length index (LI) & -0.03 & - & -0.13 & 0.12 & -0.01 & -0.09 & 0.14 & -0.08 & 0.09 & $0.23^{* *}$ & $0.31^{* *}$ & $-0.98^{* *}$ & $0.39^{* *}$ \\
\hline Thoracic index (TI) & 0.09 & -0.03 & - & $-0.52^{* *}$ & 0.11 & -0.02 & -0.03 & -0.10 & 0.03 & $-0.31^{* *}$ & -0.06 & $0.16^{*}$ & $-0.35^{* *}$ \\
\hline Depth index (DI) & 0.10 & $0.25^{* *}$ & $-0.47^{* *}$ & - & $-0.19^{*}$ & 0.08 & 0.12 & 0.13 & $0.27^{* *}$ & $0.33^{* *}$ & 0.05 & -0.14 & 0.06 \\
\hline Height index (HI) & 0.05 & -0.06 & -0.01 & -0.12 & - & $-0.16^{*}$ & 0.01 & -0.12 & $-0.32^{* *}$ & $-0.23^{* *}$ & 0.13 & 0.04 & $0.22^{* *}$ \\
\hline Thoracic development (TD) & -0.07 & $0.28^{* *}$ & -0.05 & $0.38^{* *}$ & $-0.17^{*}$ & - & $-0.82^{* *}$ & $0.96^{* *}$ & $-0.25^{* *}$ & -0.06 & $-0.91^{* *}$ & 0.13 & $-0.18^{*}$ \\
\hline Dactyl thorax index (DTI) & 0.06 & 0.09 & $0.38^{* *}$ & -0.02 & -0.05 & $-0.37^{* *}$ & - & $-0.78^{* *}$ & $0.28^{* *}$ & $0.15^{*}$ & $0.93^{* *}$ & $-0.18^{*}$ & 0.12 \\
\hline Conformation index (CI) & $0.37^{* *}$ & 0.15 & -0.001 & $0.25^{* *}$ & -0.06 & $0.81^{* *}$ & $-0.43^{* *}$ & - & $-0.36^{* *}$ & 0.05 & $-0.82^{* *}$ & 0.14 & 0.04 \\
\hline Relative cannon index (RCI) & 0.03 & $0.29^{* *}$ & $0.36^{* *}$ & $0.24^{* *}$ & $-0.16^{*}$ & $0.36^{* *}$ & $0.72^{* *}$ & $0.18^{*}$ & - & 0.10 & $-0 ; 02$ & -0.12 & -0.02 \\
\hline Index of body weight (IBW) & $0.87^{* *}$ & 0.14 & 0.07 & $0.30^{* *}$ & -0.07 & $0.27^{* *}$ & 0.08 & $0.46^{* *}$ & $0.25^{* *}$ & - & 0.15 & $-0.25^{* *}$ & $0.37^{* *}$ \\
\hline Body index (BI) & 0.04 & $0.58^{* *}$ & 0.01 & -0.11 & 0.08 & $-0.61^{* *}$ & $0.40^{* *}$ & $-0.58^{* *}$ & -0.08 & -0.10 & - & $-0.33^{* *}$ & $0.27^{* *}$ \\
\hline Proportionality (Pr) & 0.04 & $-0.99^{* *}$ & 0.03 & $-0.27^{* *}$ & 0.08 & $-0.30^{* *}$ & -0.10 & -0.15 & $-0.31^{* *}$ & -0.15 & $-0.55^{* *}$ & - & $-0.34^{* *}$ \\
\hline Area index $(\mathrm{AI})$ & $0.64^{* *}$ & $0.17^{*}$ & 0.05 & $-0.19^{*}$ & $0.21^{* *}$ & $-0.44^{* *}$ & 0.03 & 0.10 & $-0.27^{* *}$ & $0.25^{* *}$ & $0.49^{* *}$ & -0.12 & - \\
\hline
\end{tabular}

Note: ${ }^{*}(\mathrm{p}<0.05) ;{ }^{* *}(\mathrm{p}<0.01)$

Table 5. Nutritional content of forage in highland areas (Kerinci Regency and Sungai Penuh City) and lowland areas (Batanghari Regency and Muaro Jambi Regency)

\begin{tabular}{|c|c|c|c|c|c|}
\hline Location & Grass species & Crude proten $(\%)$ & Crude fiber (\%) & Ether extract (\%) & Ash (\%) \\
\hline \multirow{6}{*}{ High land } & Leersia hexandra & 9.84 & 27.97 & 1.33 & 8.30 \\
\hline & Brachiaria muticum & 10.48 & 28.00 & 1.52 & 10.77 \\
\hline & Digitaria ciliaris & 10.66 & 31.22 & 2.03 & 11.70 \\
\hline & Ottochloa nodosa & 9.74 & 31.26 & 1.37 & 10.25 \\
\hline & Echinochloa colona & 9.89 & 29.59 & 1.90 & 10.47 \\
\hline & Means & 10.12 & 29.61 & 1.63 & 10.30 \\
\hline \multirow[t]{6}{*}{ Low land } & Axonopus compresssus & 9.45 & 33.05 & 1.67 & 11.20 \\
\hline & Brachiaria muticum & 8.92 & 30.97 & 1.43 & 9.95 \\
\hline & Ottochloa nodosa & 8.38 & 30.04 & 1.40 & 10.23 \\
\hline & Leersia hexandra & 9.19 & 33.08 & 1.43 & 11.09 \\
\hline & Cynodon dactylon & 9.91 & 26.38 & 1.29 & 10.28 \\
\hline & Means & 9.33 & 30.70 & 1.44 & 10.55 \\
\hline
\end{tabular}

\section{Principal Component Analysis}

The PCA of body measurements in Thin-tail sheep revealed that Thin-tail sheep at lowland areas had 3 PC's and Thin-tail sheep at highland areas had 2 PC's (Table 6). The PCA of body measurements was accounted $72.30 \%$ in Thin-tail sheep at lowland areas and $65.84 \%$ in Thin-tail sheep at highland areas of total variance in animal morphometrics. Therefore, the PCA of body indices in Thin-tail sheep at lowland areas had 4 PC's and Thin-tail sheep at highland areas with 5 PCs (Table 7). The PCA of body indices were accounted $78.23 \%$ in Thin-tail sheep at lowland areas and $84.99 \%$ in Thin-tail sheep at highland areas of the total variance 
Table 6. Eigenvalues, total variance, cumulative, communalities, Kaiser-Meiyer-Olkin (KMO) measure of sampling adequacy and Bartlett's test of sphericity in the body measurements of Thin-tail sheep at two different areas

\begin{tabular}{|c|c|c|c|c|c|c|c|}
\hline \multirow{2}{*}{ Body measurements } & \multicolumn{4}{|c|}{ Lowland } & \multicolumn{3}{|c|}{ Highland } \\
\hline & PC1 & PC2 & PC3 & $\mathrm{EC}$ & PC1 & PC2 & $\mathrm{EC}$ \\
\hline Body weight & $0.83^{*}$ & 0.18 & 0.02 & 0.73 & $0.73^{*}$ & 0.41 & 0.70 \\
\hline Body length & $0.84^{*}$ & 0.03 & -0.10 & 0.71 & $0.73^{*}$ & 0.27 & 0.61 \\
\hline Wither height & $0.86^{*}$ & 0.17 & 0.10 & 0.78 & $0.91^{*}$ & 0.13 & 0.85 \\
\hline Chest girth & 0.16 & 0.07 & $0.92^{*}$ & 0.88 & $0.72^{*}$ & 0.30 & 0.61 \\
\hline Chest depth & $0.66^{*}$ & 0.26 & 0.06 & 0.50 & $0.49^{*}$ & 0.26 & 0.30 \\
\hline Shoulder width & 0.07 & $0.88^{*}$ & -0.06 & 0.79 & 0.27 & $0.85^{*}$ & 0.80 \\
\hline Rump height & $0.74^{*}$ & 0.25 & 0.24 & 0.66 & $0.84^{*}$ & 0.12 & 0.73 \\
\hline Rump width & 0.37 & $0.56^{*}$ & -0.52 & 0.82 & 0.12 & $0.79 *$ & 0.70 \\
\hline Cannon circumferance & 0.49 & $0.66^{*}$ & 0.27 & 0.65 & 0.34 & $0.76^{*}$ & 0.64 \\
\hline Eigenvalues & 4.18 & 1.31 & 1.02 & - & 4.72 & 1.21 & - \\
\hline Variance (\%) & 46.39 & 14.53 & 11.38 & - & 52.42 & 13.42 & - \\
\hline Cumulative (\%) & 46.39 & 60.92 & 72.30 & - & 52.42 & 65.84 & - \\
\hline KMO & \multicolumn{4}{|c|}{0.81} & \multicolumn{3}{|c|}{0.84} \\
\hline Barlett's test & \multicolumn{4}{|c|}{$* *$} & \multicolumn{3}{|c|}{$* *$} \\
\hline
\end{tabular}

Note: *main component; PC: principal component; EC: extraction communality; ${ }^{* *}(\mathrm{p}<0.01)$.

Table 7. Eigenvalues, total variance, cumulative, communalities, Kaiser-Meiyer-Olkin (KMO) measure of sampling adequacy and Bartlett's test of sphericity in the body indices of Thin-tail sheep at two different areas

\begin{tabular}{|c|c|c|c|c|c|c|c|c|c|c|c|}
\hline \multirow{2}{*}{ Body indices } & \multicolumn{5}{|c|}{ Lowland } & \multicolumn{6}{|c|}{ Highland } \\
\hline & PC1 & PC2 & PC3 & PC4 & EC & PC1 & PC2 & PC3 & PC4 & PC5 & EC \\
\hline Length index & 0.08 & $0.98^{*}$ & 0.09 & -0.02 & 0.97 & 0.11 & $0.98^{*}$ & 0.13 & -0.02 & -0.07 & 0.99 \\
\hline Thoracic index & 0.06 & -0.01 & $-0.83^{*}$ & 0.19 & 0.73 & 0.08 & -0.01 & 0.27 & 0.003 & $0.88^{*}$ & 0.85 \\
\hline Depth index & 0.01 & 0.00 & $0.75^{*}$ & 0.33 & 0.67 & 0.28 & 0.14 & 0.26 & -0.13 & $-0.81^{*}$ & 0.84 \\
\hline Height index & 0.14 & -0.04 & -0.19 & $-0.74^{*}$ & 0.61 & -0.05 & -0.11 & -0.08 & 0.45 & 0.004 & 0.22 \\
\hline Thoracic development & $-0.97^{*}$ & -0.01 & 0.02 & 0.15 & 0.96 & $0.81^{*}$ & 0.17 & 0.01 & $-0.53^{*}$ & -0.10 & 0.97 \\
\hline Dactyl thorax index & $0.96^{*}$ & 0.08 & 0.12 & 0.11 & 0.95 & -0.14 & 0.12 & $0.97^{*}$ & 0.04 & 0.04 & 0.98 \\
\hline Conformation index & $-0.92^{*}$ & -0.01 & 0.17 & 0.04 & 0.87 & $0.95^{*}$ & 0.05 & -0.01 & -0.02 & -0.01 & 0.91 \\
\hline Relative cannon index & $0.57^{*}$ & 0.15 & 0.13 & 0.56 & 0.68 & 0.24 & 0.18 & $0.92^{*}$ & -0.19 & 0.01 & 0.97 \\
\hline Index of body weight & 0.09 & 0.24 & $0.65^{*}$ & 0.17 & 0.52 & $0.65^{*}$ & 0.06 & 0.19 & 0.42 & -0.13 & 0.66 \\
\hline Body index & $0.93^{*}$ & 0.22 & 0.10 & -0.11 & 0.94 & -0.60 & $0.66^{*}$ & 0.11 & 0.42 & 0.02 & 0.98 \\
\hline Proportionality & -0.12 & $-0.97^{*}$ & -0.11 & -0.03 & 0.96 & -0.12 & $-0.97^{*}$ & -0.15 & 0.06 & 0.08 & 0.98 \\
\hline Area index & 0.11 & 0.39 & 0.47 & $-0.57^{*}$ & 0.72 & 0.02 & 0.22 & -0.02 & $0.89^{*}$ & 0.12 & 0.85 \\
\hline Eigenvalues & 4.20 & 2.50 & 1.60 & 1.26 & - & 3.18 & 2.76 & 1.68 & 1.44 & 1.13 & - \\
\hline Variance (\%) & 34.96 & 20.79 & 13.36 & 9.12 & - & 26.54 & 22.99 & 13.98 & 12.03 & 9.45 & - \\
\hline Cumulative (\%) & 34.96 & 55.75 & 69.11 & 78.23 & - & 26.54 & 49.53 & 63.61 & 75.54 & 84.99 & - \\
\hline KMO & \multicolumn{5}{|c|}{0.53} & \multicolumn{6}{|c|}{0.41} \\
\hline Barlett's test & \multicolumn{5}{|c|}{$* *$} & \multicolumn{6}{|c|}{$* *$} \\
\hline
\end{tabular}

Note: ${ }^{*}$ main component; PC: principal component; EC: extraction communality; ${ }^{* *}(\mathrm{p}<0.01)$.

in animal morphometrics. The component plot of Thintail sheep's body measurements and body indices were illustrated in Figure 4 and Figure 5.

\section{Canonical Discriminant Analysis}

The CDA revealed that one body measurement (CC) and five body indices (AI, RCI, DTI, CI, and IBW) were identified as the describing variables for Thin-tail sheep at lowland areas and highland areas (Table 8). The morphometric characterization in this study revealed moderate canonical correlation values for body measurements (0.44) and body indices (0.47). Moreover, about $67.50 \%$ of Thin-tail sheep at lowland areas and $57.50 \%$ of Thin-tail sheep at highland areas can be characterized based on body measurements (Table 9). Meanwhile, about $61.20 \%$ of Thin-tail sheep at lowland areas and $65.60 \%$ of Thin-tail sheep at highland areas can be characterized with body indices. The canonical plot of body measurements and body indices of Thintail sheep were illustrated in Figure 6.

\section{Hierarchical Cluster Analysis}

The HCA based on body weight, body measurements, and body indices were revealed two clusters 

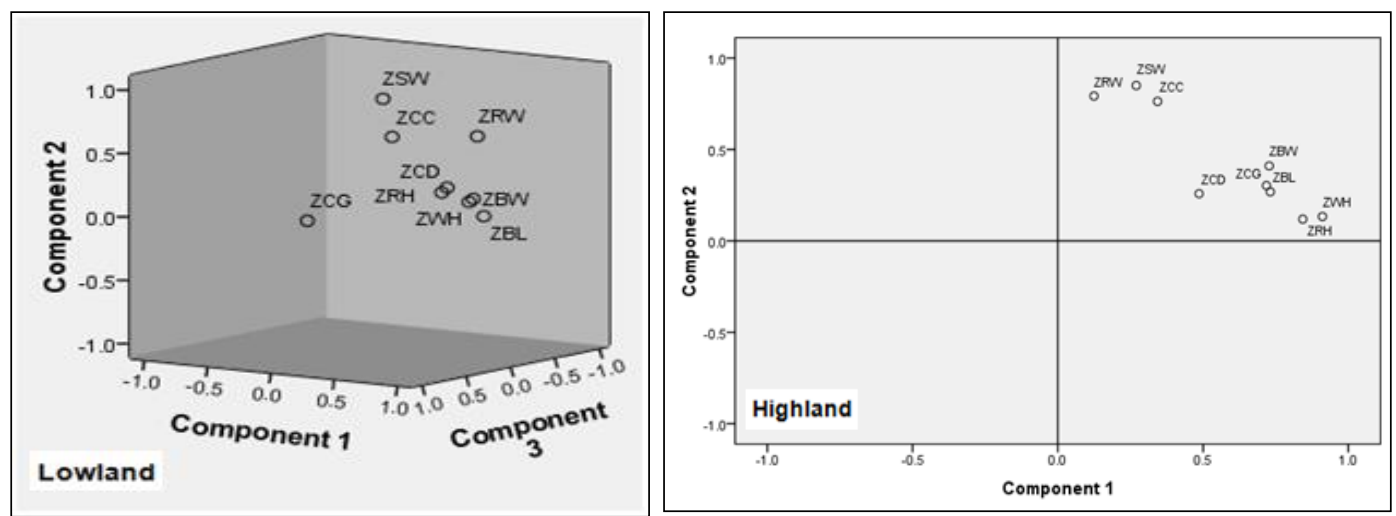

Figure 4. Component plot in rotated space for body measurements (included body weight) inThin-tail sheep
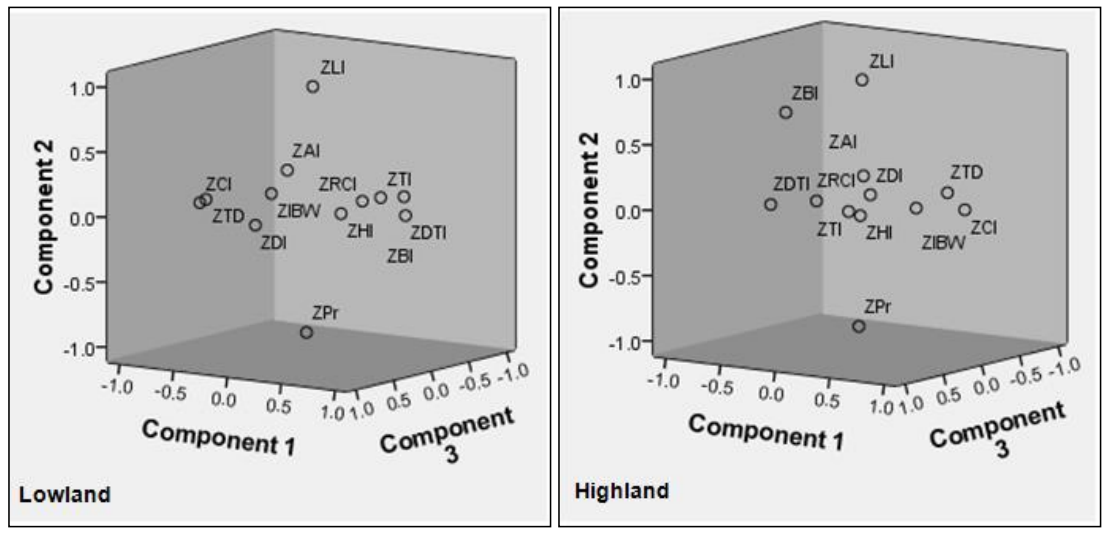

Figure 5. Component plot in rotated space for body indices in Thin-tail sheep

Table 8. Factor selected by stepwise discriminant analysis to characterize Thin-tail sheep at two different areas

\begin{tabular}{|c|c|c|c|c|c|}
\hline Factor / Step & Variables entered & Tolerance & $\mathrm{F}_{\text {remove }}$ & $\mathrm{D}^{2}$ & Wilk's $\lambda$ \\
\hline \multicolumn{6}{|c|}{ Body measurements $(\mathrm{rc}=0.44)$} \\
\hline Step 1 & Cannon circumferance & 1.00 & 76.40 & 0.96 & 0.81 \\
\hline \multicolumn{6}{|l|}{ Body indices $(\mathrm{rc}=0.47)$} \\
\hline Step 1 & Area index & 0.78 & 28.70 & 0.68 & 0.86 \\
\hline Step 2 & Relative cannon index & 0.36 & 50.59 & 0.40 & 0.91 \\
\hline Step 3 & Dactyl thorax index & 0.18 & 46.95 & 0.44 & 0.90 \\
\hline Step 4 & Conformation index & 0.30 & 28.63 & 0.68 & 0.86 \\
\hline Step 5 & Index of body weight & 0.82 & 6.73 & 0.99 & 0.80 \\
\hline
\end{tabular}

Note: $D^{2}$ : Mahalanobis distance; rc: canonical correlation.

of Thin-tail sheep at highland areas (Kerinci/KR and Sungai Penuh/SP) and Thin-tail sheep at lowland areas (Muaro Jambi/MJ and Batanghari/BA) (Figure 7). The lowest Euclidean distance showed in Kerinci-Sungai Penuh (12.70) and the highest showed in KerinciBatanghari (71.04) (Table 10). Moreover, the Euclidean distance in Muaro Jambi-Batanghari (30.33) was closed to Muaro Jambi-Sungai Penuh (32.05).

\section{DISCUSSION}

\section{Animal Morphometrics}

Table 1 shows that the temperature, humidity, rainfall, and duration of radiation in the highland areas are lower than in the lowland areas. These differences will cause differences in livestock productivity. The difference in livestock productivity is closely related to temperature and humidity factors (Rashamol et al., 2018). The interaction of temperature and humidity or "Temperature Humidity Index" (THI) can affect the comfort of life for livestock and in turn will affect the condition and productivity of livestock. High rainfall, temperature, and humidity are associated with livestock disease problems and internal and external parasites. In addition to these disease problems, many of the soil minerals are leached out during the rainy season. Nutrients are removed from the soil solution, taken by plant roots, or immobilized by microorganisms also affect the rate of percolation and leaching (de Andrade et 
Table 9. Percentage (\%) of individual classification per breed based on discriminant analysis

\begin{tabular}{|c|c|c|c|c|c|}
\hline \multirow{2}{*}{ Factor } & \multirow{2}{*}{ Variables } & \multirow{2}{*}{ Area } & \multicolumn{2}{|c|}{ Predicted group membership (N) } & \multirow{2}{*}{ Total $(\mathrm{N})$} \\
\hline & & & Lowland & Highland & \\
\hline \multirow[t]{4}{*}{ Body measurements } & Original & Lowland & $70.0(112)$ & $30.0(48)$ & $100.0(160)$ \\
\hline & & Highland & $45.5(68)$ & $57.5(92)$ & $100.0(160)$ \\
\hline & Cross-validated & Lowland & $67.5(108)$ & $32.5(52)$ & $100.0(160)$ \\
\hline & & Highland & $42.5(68)$ & $57.5(92)$ & $100.0(160)$ \\
\hline \multirow[t]{4}{*}{ Body indices } & Original & Lowland & $62.5(100)$ & $37.5(60)$ & $100.0(160)$ \\
\hline & & Highland & $33.1(53)$ & $66.9(107)$ & $100.0(160)$ \\
\hline & Cross-validated & Lowland & $61.2(98)$ & $38.8(62)$ & $100.0(160)$ \\
\hline & & Highland & $34.4(55)$ & $65.6(105)$ & $100.0(160)$ \\
\hline
\end{tabular}

Note: $\mathrm{N}=$ number of animals.
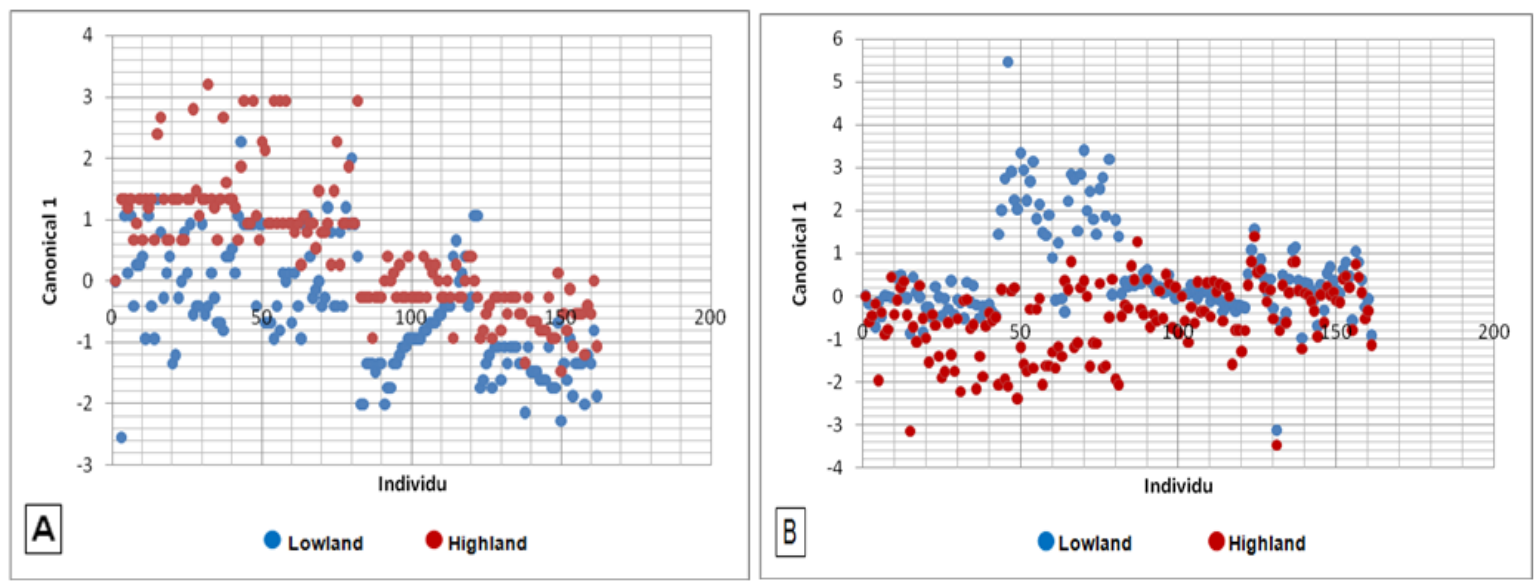

Figure 6. Canonical discriminant plot in the body measurements (A) and body indices (B) to characterize of Thin-tail sheep at two different areas

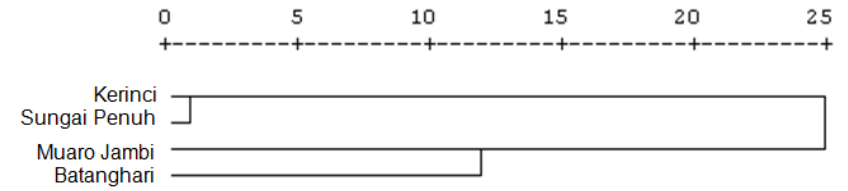

Figure 7. Dendogram distances among four different populations of Thin-tail sheep based on body weight, body measurements, and body indices.

al., 2018). Highly variable rainfall may negatively affect plant nutrient uptake and storage, potentially limiting plant growth during the growing season (Barbosa et al., 2014). Highly variable rainfall may negatively affect plant nutrient uptake and storage, potentially limiting plant growth during the growing season (Barbosa et al., 2014). Warmer regions with high temperatures are associated with lower quality forage grasses, containing higher proportions of fiber and lower proportion of crude protein, which are generally tougher to digest (Lee, 2018). Lee et al. (2017) reported that the increasing temperatures are associated with grasses of lower nutritive value, delivering higher concentrations of fibre, lower protein and lower dry matter digestibility of some forages. Argüello-Rangel et al. (2020) that forage plants in the lowlands get full sun radiation to greater the crude fiber content and lower the protein content.
Table 10. Euclidean distance between Thin-tail sheep from different populations based on body weight, body measurements, and body indices

\begin{tabular}{lcccc}
\hline Population & Kerinci & $\begin{array}{c}\text { Sungai } \\
\text { Penuh }\end{array}$ & $\begin{array}{c}\text { Muaro } \\
\text { Jambi }\end{array}$ & Batanghari \\
\hline Kerinci & 0.00 & 12.70 & 49.51 & 71.04 \\
Sungai Penuh & - & 0.00 & 32.05 & 56.38 \\
Muaro Jambi & - & - & 0.00 & 30.33 \\
Batanghari & - & - & - & 0.00 \\
\hline
\end{tabular}

Schneider et al. (2019), Sorghum plants tend to experience a decrease in crude protein as a result of increasing the intensity of sunlight. It can be noticed, especially in the plants without cutting, which developed its normal cycle, that the gross protein values decrease as the crop cycle advances. This happens because the vegetative stage has a higher leaf proportion and younger tissue since gross protein values decreased in older tissues.

The population of Thin-tail sheep at lowland areas and highland areas includes of meaty and longline types of animal. Esquivelzeta et al. (2011) reported that the average of DTI and BI in Ripollesa sheep were 9.60 \pm 0.70 and $83.90 \pm 6.10$, respectively, and included light and brevigline types of animal. Cerqueira et al. (2011) reported that the averages of DTI and BI values in Portuguese Bordaleira de Entre Douro e Minho (PBEDM) sheep 
were 8.88-9.84 and 80.37-84.25, respectively, and included light and brevigline types of animal. In addition, according to DTI value, the Pramenka sheep included light type of animal (DTI= 8.05-9.31) as reported by Markovic et al. (2019). The BW of Thin-tail sheep in this study was lower than those of Ripollesa (51.40 \pm 6.63 $\mathrm{kg})$, Djallonke $(21.70 \pm 0.50 \mathrm{~kg})$, Sahel $(27.50 \pm 0.80 \mathrm{~kg})$, Zulu $(34.70 \pm 0.20 \mathrm{~kg})$, Morada Nova $(31.74 \pm 5.09 \mathrm{~kg})$, Balami $(60.87 \pm 14.31 \mathrm{~kg})$, Koroji $(64.12 \pm 16.42 \mathrm{~kg})$, Uda $(57.83 \pm 17.76 \mathrm{~kg})$, Yankasa $(51.09 \pm 8.47 \mathrm{~kg})$, Sohagi $(48.20 \pm 1.73 \mathrm{~kg})$, Rampur-Bushair $(25.72 \pm 0.16 \mathrm{~kg})$, Istrian Pramenka (61.29 $\pm 0.90 \mathrm{~kg})$, and PBEDM (32.92 \pm 5.05 $\mathrm{kg}$ ) sheep (Esquivelzeta et al., 2011; Birteeb et al., 2012; Mavule et al., 2013; Silva et al., 2015; Dauda et al., 2018; Elsaid \& Elnahas, 2019; Sankhyan et al., 2018; Markovic et al., 2019; Cerqueira et al., 2019). Purwanti et al. (2019) reported body index of Ettawa Grade does of Indonesia did not affect the litter size. The results of the study, chest girth significantly affects $(\mathrm{p}<0.05)$ body weight. The variation in BW could be due to breeds, management, or the environment. The productivity of sheep is affected by many factors, such as breed (genetic) and environmental factors (Petrovic et al., 2011).

\section{Phenotypic Correlation}

The correlation value between BW and CG in Thintail sheep at highland areas included of the high category $(0.61<\mathrm{r}<0.80)$ and similar to Uda $(0.74-0.83)$ and Zulu (0.74) sheep (Yakubu \& Akinyemi, 2010; Mavule et al., 2013). Moderate category of $\mathrm{r}$ value $(0.40<\mathrm{r}<0.60)$ between BW and CG were reported in Rampur-Bushair (0.47) and Sohagi (0.55) sheep (Sankhyan et al., 2018; Elsaid \& Elnahas, 2019). Very high category of $r$ value $(0.81<\mathrm{r}<1.00)$ between BW and CG were reported in Morada Nova (0.93) and Pramenka (0.87) sheep (Silva et al., 2019; Markovic et al., 2019). The different results of this study compared to previous studies can be caused by genetic (breed), management, and environmental factors. Indriani et al. (2020) reported that the cellulose and hemicellulose contents of grass at highland areas were lower than in lowland areas $(\mathrm{p}<0.05)$. Moreover, the fat deposit in sheep in lowland areas was lower than in sheep in highland areas because of high temperatures. Less fat deposit in sheep in lowland areas is important to reduce body temperature. Therefore, a low correlation between BW and CG in sheep in lowland areas may be caused by less fat deposit. Other factors that cause differences in the characteristics of sheep at the highland and lowland areas were the species and quality of forage consumed by the sheep. Forage in the lowlands will undergo a ripening process earlier than in the highlands, causing a decrease in forage quality due to an increase in cell walls, which are the main component of crude fiber. The crude fiber content in mature plants tends to increase, even in certain plants, it exceeds the increase in crude fiber in other plants (Nelson \& Moser, 1994). Moreover, the fat deposit in lowland sheep was lower than in highland sheep because of the high temperature. Thus, less fat deposit in the lowland sheep is important to reduce body temperature. So, a low cor- relation between BW and CG in lowland sheep may be caused by less fat deposit.

Table 5 shows that the quality of forage consumed by thin-tail sheep in the lowland areas is lower than that of forage consumed by thin-tail sheep in the highland areas. The averages forage CP and EE contents consumed by sheep in the highland areas are higher than those consumed by the thin-tail sheep in the lowland areas. In contrast, thin-tail sheep's CF and Ash contents at highland areas are lower than those at the lowland areas. Turral et al. (2011) stated that the temperature was higher in the lowlands and medium areas, resulting in higher cellulose and hemicellulose content than in the highlands area. Plants in the highland areas develop with lower light intensity and air temperature so that protein quality is higher and is inversely proportional to its fiber content. Rochana et al. (2016) stated that forage plants in the lowlands get full sun radiation to greater the crude fiber content. Furthermore, it was also stated that the content of NDF, cellulose, and hemicellulose in the lowland areas and medium-land areas were higher than in the highland areas (Indriani et al., 2020). The higher the increase in crude fiber content, the higher the decrease in nutritional value (Setiyaningrum et al., 2018). Crude fiber content will affect feed digestibility in ruminants. The higher the crude fiber content, the lower the feed digestibility (Rustiyana et al., 2016). Based on the description above, it can be stated that the altitude influences the quality of forage.

\section{Principal Component Analysis}

The PCA of body measurements in Thin-tail sheep revealed that thin-tail sheep at lowland areas had 3 PC's and thin-tail sheep at highland areas had 2 PC's (Table 4). The PCA of body measurements was accounted to $72.30 \%$ in thin-tail sheep at lowland areas and $65.84 \%$ in thin-tail sheep at highland areas of the total variance in animal morphometrics. The determinant of body sizes that significantly different $(\mathrm{P}<0.01)$ and the highest in thin-tail sheep at the lowland areas and highland areas is the withers height, while the determinant of the shape in the thin-tail sheep at highland areas and lowland areas is shoulder width. Therefore, the PCA of body indices in Thin-tail sheep at lowland areas had 4 PC's and thin-tail sheep at highland areas with 5 PCs (Table 5). The PCA of body indices were accounted $78.23 \%$ in thin-tail sheep at lowland areas and $84.99 \%$ in thin-tail sheep at highland areas of the total variance in animal morphometrics. Previous studies obtained PCA of body measuements with accounting in total variance of about $59.00 \%$ (with 2 PC's) in Ripollesa sheep (Esquivelzeta et al., 2011); 70.47\% (with 2 PC's) in PBEDM sheep (Cerqueira et al., 2011); 87.19\% (with 2 PC's) in Ghanaian sheep (Birteeb et al., 2012); 62.13\% (with 4 PC's) in Zulu sheep (Mavule et al., 2013); 75.21\% (with 3 PC's) in Yankasa sheep (Yakubu, 2013); 66.91\% (with 2 PC's) in Balami sheep (Yunusa et al., 2013); 57.43\% (with 2 PC's) in Uda sheep (Yunusa et al., 2013); 72.28\% (with 3 PC's) in Morada Nova sheep (Silva et al., 2015); 61.53\% (with 4 PC's) in Rampur-Bushair sheep (Sankhyan et 
al., 2018); $57.80 \%$ (with 2 PC's) in Sohagi sheep (Elsaid \& Elnahas, 2019); 96.65\% (with 3 PC's) in Pramenka sheep (Markovic et al., 2019), and 68.04\% (with 3 PC's) in Djallonke sheep (N'Goran et al., 2019). Unfortunately, the study of the PCA of body indices so far has not been reported. However, previous studies reported that PCA of body indices was accounted $86.84 \%$ (with 4 PC's) in Katjang does and $89.38 \%$ (with 4 PC's) in Pasundan cow (Putra \& Ilham, 2019; Putra et al., 2020). Malewa et al. (2008) reported the main characteristic variable of body size for local hammer sheep was chest circumference. Furthermore, Gunawan et al. (2011) also reported the characteristic measure that has a positive correlation with the size score is the chest circumference of all types of Indonesian Garut sheep. This study indicates that CG can be used as a selection parameter to increase the body size score of Thin-tail sheep both in the highland areas and lowland areas in Jambi Province.

The KMO values in PCA of body measurements were higher than PCA of body indices. According to the KMO value, PCA of body measurements in Thintail sheep at lowland areas and highland areas were accurate $(\mathrm{KMO}>0.50)$. Hence, the KMO values in PCA of body indices in Thin-tail sheep at the highland area were not accurate $(\mathrm{KMO}<0.50)$. The component plots of Thin-tail sheep's body measurements and body indices were illustrated in Figure 4 and Figure 5, respectively. Garut sheep is one Indonesian native Thin-tail sheep with registered breeds standard (SNI) based on age, $\mathrm{BW}, \mathrm{WH}, \mathrm{BL}, \mathrm{CG}$, and scrotum circumference (for male). Those parameters were classified into the first component for lowland and highland sheep.

\section{Canonical Discriminant Analysis}

Previous studies reported that the body measurements could be used to characterize $70 \%-100 \%$ of Ripollesa sheep at nine different populations (Esquivelzeta et al., 2011); 54.18\%-74.59\% of Algerian sheep at three different populations (Dekhili, 2014) and $76.27 \%-92.80 \%$ of Djallonke ewes at three different populations (N'Goran et al., 2019). Moreover, the body measurements were used by Asamoah-Boaheng \& Sam (2016), reporting that $65.20 \%$ of Djallonke sheep, $88.90 \%$ of Sahel sheep, and $79.30 \%$ of Djallonke $\times$ Sahel sheep can be characterized with body measurements. The classification percentage in this study was lower than previous studies and needed more morphometric measurements. The cephalic measurements are important to obtain CDA. Popoola \& Oseni (2018) obtained four cephalic measurements of skull width, head width, head length, and head depth as the describing variable to characterize four Nigerian sheep breeds. Moreover, Asamoah-Boaheng \& Sam (2016) obtained head width and face length as the describing variable in three African sheep breeds. Meanwhile, Nunes et al. (2020) reported that about $57.1 \%$ (kept at Ceara region) and $54.5 \%$ (kept at Rio Grande do Norte region) of Marada Nova sheep could be classified into the original population based on their body indices. The difference in body measurements, presumably due to the differences in the environment, especially altitude. Indriani et al. (2020) stated that the content of NDF, cellulose, and hemicellulose in the lowlands and medium areas is higher than in the highlands. Increasing crude fiber content will reduce the nutritional value (Setiyaningrum et al., 2018). Crude fiber content will affect the feed digestibility in ruminants. The higher crude fiber content of feed will reduce the feed digestibility (Rustiyana et al., 2016). This study's rc value included moderate category and reveals that about $40 \%$ of lowland sheep and highland sheep can be classified with body measurements or body indices. Therefore, about $60 \%$ of both sheep may be classified by other factors such as phenotypic traits and genetic diversity.

\section{Hierarchical Cluster Analysis}

The HCA based on body weight, body measurements, and body indices revealed two clusters of highland clusters (Kerinci/KR and Sungai Penuh/SP Regencies) and two clusters of lowland clusters (Muaro Jambi/MJ and Batanghari/BA Regencies) as were illustrated in Figure 6. The lowest Euclidean distance showed in Kerinci-Sungai Penuh (12.70) and the highest showed in Kerinci-Batanghari (71.04), as presented in Table 8. Moreover, the Euclidean distance in Muaro Jambi-Batanghari (30.33) was closed to Muaro JambiSungai Penuh (32.05). Previous studies obtained the Euclidean distance of 1.87 to 8.87 among Pramenka sheep from six different populations in Slovenia (Markovic et al., 2019) and 2.34 to 7.77 among Black Creole goats from seven different populations in Mexico (Jarquin et al., 2019). Therefore, Handiwirawan et al. (2011) obtained the Euclidean distance of 10.83 to 91.39 among five sheep breeds. Despite with morphometric, the phylogeny tree in the sheep can be obtained based on bloods profile (Geng et al., 2003), mitochondrial DNA diversity (Mariotti et al., 2013), and microsatellite locus (Jakaria et al., 2012). The difference in animal management, selection, geographical area, agro-climatic conditions, and natural resources can affect the phenotypic variation in a breed in each population.

\section{CONCLUSION}

Five body measurements (BW, BL, WH, CD, and $\mathrm{RH}$ ) and two body indices (TD and $\mathrm{CI}$ ) were identified as the first component for explaining sheep morphometrics at lowland areas and highland areas. Around 67.5\% of thin-tail sheep at lowland areas and $57.5 \%$ of thin-tail sheep at highland areas can be characterized with body measurements. Hence, about $61.2 \%$ thin-tail sheep at lowland areas and $65.6 \%$ thin-tail sheep at highland areas can be characterized with body indices. The cluster analysis in four Thin-tail populations revealed two clusters, i.e., cluster 1 (Kerinci and Sungai Penuh) and cluster 2 (Muaro Jambi and Batanghari). Around 60\% of Thin-tail sheep can be characterized through their body indices to be used as a reference for selection and sheep breeding programs in the future. 


\section{CONFLICTS OF INTEREST}

The authors declare that they have no conflicts of interest.

\section{ACKNOWLEDGEMENT}

This work was funded by DIPA PNPB Faculty of Animal Science of Jambi University, Indonesia (grant number 269/UN21.17/PP/2017).

\section{REFERENCES}

Argüello-Rangel, J., L. Mahecha-Ledesma, \& J. AnguloArizala. 2020. Nutritional and productive profile of shrub species in tropical lowlands of Antioquia (Colombia). Ciencia y Tecnología Agropecuaria 21:e1700. https://doi. org/10.21930/rcta.vol21_num3_art:1700

Asamoah-Boaheng, M. \& E. K. Sam. 2016. Morphological characterization of breeds of sheep: a discriminant analysis approach. Springer Plus 5: 1-12. https://doi.org/10.1186/ s40064-016-1669-8

Ashari, M., R. R. A. Suhardiani, \& R. Andriati. 2015. Performance of the body weight and the linear body size of the certain age of fat-tailed sheep in east Lombok subdistricts. Jurnal Ilmu dan Teknologi Peternakan Indonesia 1: 24-30.

Ashifudin, M., E. Kurnianto, \& Sutopo. 2017. Morphometrical characteristics of red comb and black comb Kedu chicken of first generation in satker ayam Maron-Temanggung. Jurnal Ilmu Ternak. 17: 40-46. https://doi.org/10.24198/jit. v17i1.14825

Aziz M. M. A. \& F. S. Al-Hur. 2013. Differentiation between three Saudi sheeps types using size-free canonical discriminant analysis. Emir. J. Food Agric. 25:723-735. https://doi. org/10.9755/ejfa.v25i9.15827

Barbosa, E. R. M., K. W. Tomlinson, L. G. Carvalheiro, K. Kirkman, S. de Bie, H. H. T. Prins, \& F. van Langevelde. 2014. Short-term effect of nutrient availability and rainfall distribution on biomass production and leaf nutrient content of savanna tree sSpecies. PLoS ONE 9: e92619. https:// doi.org/10.1371/journal.pone.0092619

Birhanie, M., K. Alemayehu, \& G. Mekuriaw. 2019. Morphological characterization of goat populations in Central Zone of Tigray, Ethiopia. Trop. Ani. Sci. J. 42:8189. https://doi.org/10.5398/tasj.2019.42.2.81

Birteeb, P. T., O. P. Sunday, A. Yakubu, M. A. Adeleke, \& M. O. Ozoje. 2013. Multivariate characterisation of the phenotyphic traits of Djallonke and Sahel sheep in Northern Ghana. Trop. Anim. Health. Prod. 45: 267-274. https://doi. org/10.1007/s11250-012-0211-4

Birteeb, P. T., O. P Sunday, \& O. O. Michael. 2014. Analysis of the body structure of Djallonke sheep using multideterminant approach. Anim. Genet. Res. 54: 65-72. https://doi. org/10.1017/S2078633614000125

Boujenane, I. 2015. Multivariate characterisation of OulmesZaer and Tidili cattle using the morphological traits. Iranian J. Appl. Anim. Sci. 5: 293-299.

Boujenane, I., L. Derqaoui, \& G. Nouamane. 2016. Morphological differentiation between two Maroccan sheeps breeds. J. Livest. Sci. Tech. 4: 31-38.

Ceccobelli, S., P.D. Lorenzo, F. Panella, E. Lasagna, \& F.M. Sarti. 2016. Morphological and genetic characterisation of Pagliarola breed and its genetic relationships with other three indigenous Italian sheep breeds. Ital. J. Anim. Sci. 15: 47-54. https://doi.org/10.1080/1828051X.2016.1139325

[Central Bureau of Statistic, Jambi Province]. 2021. Jambi Province in Numbers. Central Bureau of Statistic, Jambi
Province. CV. Dharmaputra, Jambi.

Cerqueira, J. O. L., X. Feas, A. Iglesias, L. F. Pacheco, \& J. P. P. Araujo. 2011. Morphological traits in Portuguese Bordaleira de Entre Douro e Minho sheep: divergence of the breed. Anim. Prod. Sci. 51: 635-641. https://doi. org/10.1071/AN10147

Chacon, E., F. Macedo, F. Velazquez, S. R. Paiva, E. Pineda, \& C. McManus. 2011. Morphological measurements and body indices for Cuban Creole sheeps and heir crossbreds. Rev. Bras. Zootec. 40: 1671-1679. https://doi.org/10.1590/ S1516-35982011000800007

Dauda, A., H. Y. Abbaya, \& V. N. Ebegbulem. 2018. Application of multifactorial discriminant analysis of morphostructural differentiation of sheep. J. Genet. Gen. Eng. 2 (2):11-16.

de Andrade, C. W. L., S. M. G. L. Montenegro, J. H. de Miranda, A. A. de A. Montenegro, \& F. M. C. V. de Assis. 2018. Simulation of sodium and potassium dynamics by the hydrus $2 \mathrm{~d}$ model in a Haplic Planosol via residue water. Engenharia Agrícola, Jaboticabal, 38:874-884. https:// doi.org/10.1590/1809-4430-Eng.Agric.v38n6p874-884/2018

Dekhili, M. 2014. A morphometric study of sheep reared in North-East Algerian. Arch. Zootec. 63: 623-631. https://doi. org/10.4321/S0004-05922014000400006

Elsaid, R. \& A. Elnahas. 2019. Principal component analysis of body measurements of Sohagi sheep in Upper Egypt. Egypt. J. Sheep. Sheeps. Sci. 14: 83-92.

Esquivelzeta, C., M. Fina, R. Bach, C. Madruga, G. Caja, J. Casellas, \& J. Piedrafita. 2011. Morphological analysis and subpopulation characterization of Ripollesa sheep breed. Anim. Genet. Res. 49: 9-17. https://doi.org/10.1017/ S2078633611000063

[FAO] Food and Agriculture Organization. 2012. Phenotypic Characterization of Animal Genetic Resources. FAOAnimal Production and Health Guidelines No. 11. Commission on Genetic Resources for Food and Agriculture. Rome, Italy.

Gatew, H., H. Hassen, K. Kebede, A. Haile, R.N.B. Lobo, A. Yetayew, \& B. Rischkowsky. 2015. Husbandry practices and phenotypic characteristics of indigenous goat populations in Ethiopia. African Journal of Agricultural Research 12: 2729-2741. https://doi.org/10.5897/AJAR2016.11282

Getahun, S., S Ahmed, \& W. Zemene. 2020. Morphometric characterization of indigenous Goats in East Gojjam Zone, Amhara Region, Ethiopia. Int. J. Adv. Res. Biol. Sci. 7: 47-62.

Geng, R. Q., H. Chang, Z. P. Yang, W. Sun, L. P. Wang, S. X. Lu, K. Tsunoda \& Z. J. Ren. 2003. Study on origin and phylogeny status Hu sheep. Asian-Aust. J. Anim. Sci. 16: 743-747. https://doi.org/10.5713/ajas.2003.743

Gunawan, A., R. Mulyono, \& C. Sumantri. 2011. Identification of body size and body shape of Garut sheep fighting type and meat type and Garut cross based on principal component analysis. Animal Production 11: 8-14.

Handiwirawan, E., R. R. Noor, C. Sumantri \& Subandriyo. 2011. The differentiation of sheep breed based on the body measurements. J. Indonesian Trop. Anim. Agric. 36: 1-8. https://doi.org/10.14710/jitaa.36.1.1-8

Hilmawan F., H. Nuraini, R. Priyanto, \& B. W. W. Putra. 2017. Morphometric measurement of male Ongole crossbred cattle and Buffalo by digital image analysis. Jurnal Veteriner 15: 587-596. https://doi.org/10.19087/ jveteriner.2016.17.4.587

Hosseini, M., H. M. Shahrbabak, M. B. Zandi, \& M. H. Fallahi. 2016. A Morphometric survey among three Iranian horse breeds with multivariate analysis. Med. Pet. 39:155-160. https://doi.org/10.5398/medpet.2016.39.3.155

Indriani, N. P., A. Rochana, H. K. Mustafa, B. Ayuningsih, I. Hernaman, D. Rahmat, T. Dhalika, K. A. Kamil, \& Mansyur. 2020. The Effect of various altitudes on field grass forage fiber fraction content. Jurnal Sain Peternakan 
Indonesia 15: 212-218. https://doi.org/10.31186/jspi. id.15.2.212-218

Jakaria, M. S. A. Zein, S. Sulandari, Subandriyo, \& Muladno. 2012. The use of microsatellite markers to study genetic diversity in Indonesian sheep. J. Indonesian Trop. Anim. Agric. 37: 1-9. https://doi.org/10.14710/jitaa.37.1.1-9

Jarquin, J. C. S., S.I.R. Ponce, M.D. Aguilar, H. R. V. Avila, V. H. C. Sandoval, \& H. M. A. Montemayor. 2019. Morphostructural characterization of the Black Creole sheeps raised in Central Mexico, a currently threatened zoogenetic resource. Animals 9: 1-12. https://doi.org/10.3390/ ani 9070459

Josiane M., H. Gilbert, \& D. Johann. 2020. Genetic parameters for growth and kid survival of indigenous Goat under smallholding system of Burundi. Animals 10: 1-10. https:// doi.org/10.3390/ani10010135

[KEMENTAN RI] Ministry of Agriculture Indonesia. 2017. Livestock and Animal Health Statistics 2017. Directorate General of Animal Livestock and Health. Jakarta, Indonesia.

Khargharia, G., G. Kadirvel, \& S. Kumar. 2015. Principal component analysis of morphological traits of Assam hill sheeps in Eastern Himalayan India. J. Anim. Plant. Sci. 25: 1251-1258.

Lee, M. A. 2018. A global comparison of the nutritive values of forage plants grown in contrasting environments. Journal of Plant Research 131:641-654. https://doi.org/10.1007/ s10265-018-1024-y

Lee, A. M., A. P. Davis, M. G. G. Chagunda, \& P. Manning. 2017. Forage quality declines with rising temperatures, with implications for livestock production and methane emissions. Biogeosciences 14: 1403-1417. https://doi. org/10.5194/bg-14-1403-2017

Mahmudi, R. Priyanto, \& Jakaria. 2019. Morphometric characteristics of Aceh, Ongole crossbreed and Bali cattle based on PCA. Journal of Animal Production and Processing Technology 07: 35-40. https://doi.org/10.29244/7.1.35-40

Malewa, A. D. \& Salmin. 2008. The characteristics of Palu local sheep based on morphometric diversity. J. Agroland. 15: 68-74.

Mariotti, M., A. Valentini, A., P. A. Marsan \& L. Pariset. 2013. Mitochondrial DNA of seven Italian sheep breeds shows faint signatures of domestication and suggests recent breed formation. Mitochondrial DNA 24: 577-583. https:// doi.org/10.3109/19401736.2013.770493

Markovic, B., P. Dove, M. Markovic, D. Radonjic, M. Adakalic, \& M. Simcic. 2019. Differentiation of some Pramenka sheep breeds based on morphometric characteristics. Archives Anim. Breed. 62: 393-402. https://doi.org/10.5194/ aab-62-393-2019

Mavule, B. S., V. Muchenje, C. C. Bezuidenhout, N. W. Kunene. 2013. Morphological structure of Zulu sheep based on principal componentanalysis of body measurements. Small Rum. Res. 111: 23-30. https://doi.org/10.1016/j. smallrumres.2012.09.008

Mulyono, R. H., C. Sumantri, R. R. Noor, Jakaria, \& D. A. Astuti. 2018. The prediction of prolificacy using linear body parameters and craniometric analysis in EtawahGrade does. Trop. Anim. Sci. Journal. 41:77-84. https://doi. org/10.5398/tasj.2018.41.2.77

Nafti, M., Z. Khaldi, \& B. Haddad. 2014. Multivariate characterization of morphological traits in local Tunisian oases sheepss. Anim. Genet. Res. 55 :29-38. https://doi. org/10.1017/S2078633614000265

Najmuddin, M. \& M. Nasich. 2019. Thin tailed ewe productivity in Sedan Village, Sedan District, Rembang Sub-districts. Journal of Tropical Animal Production 20: 76-83. https:// doi.org/10.21776/ub.jtapro.2019.020.01.10

N'Goran, K. E., K. G. Severin, K. N. Cyrille, L. N. Etienne,
\& E. J. Yves. 2019. Primary morphological characterization of West African dwarf (Djallonke) ewes from Cote d'Ivoire based on qualitative and quantitative traits. Int. J. Genet. Mol. Biol. 11: 16-28. https://doi.org/10.5897/ IJGMB2019.0170

Nunes, S. F., J. Ferreira, R.M.F. Silveira, D.C. Sales, J.E.R. de Sousa, S.R. Paiva, \& D.A.E. Facanha. 2020. Morphometric characterization and zoometric indices of white Morada Nova breed: The first step for conservation. Small Rum. Res. 192: 106178. https://doi.org/10.1016/j. smallrumres.2020.106178

Nelson, C. J., \& L. E. Moser. 1994. Plant Factors Affecting Forage Quality. In: G.C.J. Fahey, L.E. Moser, D.R. Martens, \& M. Collins. (Eds.) Forage Quality, Evaluation, and Utilization, American Society of Agronomy Crop Science Society of America Soil Science Society of America, Madison. Pp.115154. https://doi.org/10.2134/1994.foragequality.c3

Oliveira, E. J., R. P. Savegnano, L. A. de Freitas, A. P. Freitas, S. R. Maia, F. F. Simili, L. E. Faro, R. L. D. da Costa, M. L. S. Junior, \& C. C. P. de Paz. 2018. Estimates of genetic parameters and cluster analysis for worm resistance and resilience in Santa Ines meat sheep. Pesq. Agropec. Braz. 53: 13381345. https://doi.org/10.1590/s0100-204x2018001200006

Ouchene-Khelifi, N. A., N. Ouchene, A. D. Silva, \& M. Lafri. 2018. Multivariate characterization of phenotypic traits of Arabia, the main Algerian sheeps breed. Livest. Res. Rur. Dev. 30: 116.

Petrovic, M. P., D. R. Muslic, V. C. Petrovic, \& N. Maksimovic. 2011. Influence of environmental factors on birth weight variability of indigenous Serbian breeds of sheep. Afr. J. Biotechnol. 10: 4673-4676.

Popoola, M. A. \& S. O. Oseni. 2018. Multifactorial discriminant analysis of cephalic morphology of indigenous breeds of sheep in Nigeria. Slovak J. Anim. Sci. 51: 4551.

Purwanti, D., E. T. Setiatin, \& E. Kurnianto. 2019. The morphometric performance of Ettawa Grade goat in various parity in Center for Integrated Livestock Breeding and Management in Kendal Sub-districts. Jurnal IlmuIlmu Peternakan 29: 15-23. https://doi.org/10.21776/ ub.jiip.2019.029.01.03

Putra, W. P. B. \& F. Ilham. 2019. Principal component analysis of body measurements and body indices and their correlation with body weight in Katjang does of Indonesia. J. Dairy. Vet. Anim. Res. 8: 124-134. https://doi.org/10.15406/ jdvar.2019.08.00254

Putra, W. P. B., S. Said, \& J. Arifin. 2020. Principal component analysis (PCA) of body measurements and body indices in the Pasundan cows. BSJ Agri. 3: 49-55.

Rashamol, V.P., V. Sejian, M. Bagath, G. Krishnan, P. R. Archana, \& R. Bhatta. 2018. Physiological adaptability of livestock to heat stress: an updated review. J. Anim. Behav. Biometeorol. 6: 62-71. https://doi.org/10.31893/23181265jabb.v6n3p62-71

Rochana, A., N. P. Indriani, B. Ayuningsih, I. Hernaman, T. Dhalika, D. Rahmat, \& S. Suryanah. 2016. Feed forage and nutrition value at altitudes during the dry season in West Java. Animal Production 18: 85-93. http://dx.doi. org/10.20884/1.anprod.2016.18.2.531

Rustiyana, E., Liman, \& F. Fathul. 2016. Effect of substitution of elephant grass (Pennisetum purpureum) with palm leave sheat on the digestibility of crude protein and crude fiber digestibility in goats). Jurnal Ilmiah Peternakan Terpadu. 4: 161-165.

Sabbioni, A., V. Beretti, P. Superchi, \& M. Ablondi. 2020. Body weight estimation from body measures in Cornigliese sheep breed. Ital. J. Anim. Sci. 19: 25-30. https://doi.org/10. 1080/1828051X.2019.1689189

Salvagno, L. \& U. Albarella. 2017. A morphometric system to distinguish sheep and goat postcranial bones. Plos One. 
12: 1-37. https://doi.org/10.1371/journal.pone.0178543

Sanni, M.T., M. Okpeku, G. O. Onasanya, M. A. Adeleke, M. Wheto, A S. Adenaike, B. O. Oluwatosin, O. A. Adebambo, \& C. O. N. Ikeobi. 2018. Genetic morphometry in nigerian and south african kalahari red goat breeds. Agricultura Tropica Et Subtropica 51:51-61. https://doi. org/10.2478/ats-2018-0006

Sankhyan, V., Y. P. Thakur, S. Katoch, P. K. Dogra, \& R. Thakur. 2018. Morphological structuring using principal component analysis of Rampur-Bushair sheep under transhumance production in western Himalayan region,India. Indian J. Anim. Res. 52:917-922. https://doi. org/10.18805/ijar.B-3296

Saputra, D.A., Maskur, \& T. Rozi. 2019. Morphometric characteristics (linear size and body circle) of Bali cattle that are raised semi-intensively in Sumbawa Sub-districts. Indonesian Journal of Animal Science and Technology 5: 67-75. https://doi.org/10.29303/jitpi.v5i1.53

Schneider, J. R , B. O. Caron, E. F. Elli, F. Schwarze, \& T. D. Engroff. 2019. Solar radiation use efficiency and gross protein of sorghum forage are modified by the cutting management. Ciência Animal Brasileira 20:1-13. https://doi. org/10.1590/1809-6891v20e-46662

Setiyaningrum, E., I N. Kaca, \& N. K. E. Suwitari. 2018. The effect of cutting age on production and nutritional quality of indigofera plants (Indigofera Sp). Gema Agro. 23:59-62. https://doi.org/10.22225/ga.23.1.660.59-62

Silva, M. S., L. Shiotsuki, R. N. B. Lobo, \& O. Faco. 2015. Principal component analysis of for evaluating a ranking method used in the performance testing in sheep of Morada Nova breed. Cienc. Agr. 36:3909-3922. https://doi. org/10.5433/1679-0359.2015v36n6p3909

Traore, A., H. H. Tamboura, A. Kabore, L. J. Royo, I. Fernandez , I. Alvarez, M. Sangare, D. Bouchel, J. P. Poivey, D. Francois, L. Sawadogo, \& F. Goyache. 2008. Multivariate analyses on morphological traits of sheepss in Burkina Faso. Arch. Tierz. 6:588-600. https://doi.org/10.5194/ aab-51-588-2008

Turral, H., J. Burke, \& J.M. Faures. 2011. Climate change, water and food security. FAO. Water Respons. Rome. 27.

Vazic, B., B. Rogic, I. Pihler, M. Drinić, \& N. Savić. 2017. Morphometric characterization and body measurement correlation in dubska pramenka sheep. Contemporary Agriculture. 66:38-43. https://doi.org/10.1515/ contagri-2017-0007

Yakubu A. 2013. Pincipal component analysis of the conformation traits of Yankasa sheep. Biotechnol. Anim. Husb. 29:65-74. https://doi.org/10.2298/BAH1301065Y

Yakubu, A. \& M. O. Akinyemi. 2010. An evaluation of sexual size dimorphism in Uda sheep using multifactorial discriminant analysis. Acta Agric. Scand Sect. 60:74-78. https://doi.org/10.1080/09064702.2010.502242

Yunusa, A. J., A. E. Salako, \& O. A. Oladejo. 2013. Principal component analysis of he morphostructure of Uda and Balami sheep of Nigeria. Int. Res. J. Agric. Sci. 1:45-51.

Zaitoun, I. S., M. J. Tabbaa, \& S. Bdour. 2005. Differentiation of native sheeps breeds of Jordan on the basis of morphostructural characteristics. Small Rum Res. 56:173-182. https://doi.org/10.1016/j.smallrumres.2004.06.011 